\title{
EFFECT OF WIND LOAD ON TALL BUILDINGS IN DIFFERENT TERRAIN CATEGORY
}

\author{
Mohammed Asim Ahmed ${ }^{1}$, Moid Amir ${ }^{2}$, Savita Komur ${ }^{3}$, Vaijainath Halhalli ${ }^{4}$ \\ ${ }^{1}$ Students, Dept.of civil engineering, PDA College of engineering, Gulbarga, Karnataka, India \\ ${ }^{2}$ Students, Dept.of civil engineering, PDA College of engineering, Gulbarga, Karnataka, India \\ ${ }^{3}$ Students, Dept.of civil engineering, PDA College of engineering, Gulbarga, Karnataka, India \\ ${ }^{4}$ Associate Professor, Dept.of civil engineering, PDA College of engineering, Gulbarga, Karnataka, India
}

\begin{abstract}
Wind is a perceptible natural motion of air relative to earth surface, especially in the form of air current blowing in a particular direction. The major harmful aspect which concern to civil engineering structures is that, it will load any and every object that comes in its way. Wind blows with less speed in rough terrain and higher speed in smooth terrain. This paper presents displacement accour in different storey due to wind in different terrain category. Three models are analyse using ETABS 2015 package. Present works provides a good source of information about variation in deflection as height of model changes and percentage change in deflection of same model in different terrain category.
\end{abstract}

Keywords: TG-1, TG-2, TG-3, TG-4, ETABS 2015, Deflection, \% ${ }^{\text {age }}$ of deflection

\section{INTRODUCTION}

High rise building means the building are tall say, "more than twelve storeys" or, high-rise building is defined as a structure "if height more than 35 meter" says tall building. The wind flow interacts only with the external shape of the structure for static structure. For dynamic structures there is an additional interaction with the motion of the structure. The average wind speed over a time period of the order of ten minutes or more, tends to increase with height, while the gustiness tends to decrease with height.

Wind blows with less speed in rough terrain and higher speed in smooth terrain. Terrain in which a specific structure stands shall be assessed as being one of the following terrain categories:

Category 1 - Exposed open terrain with few or no obstructions and in which the average height of any object surrounding the structure is less than $1.5 \mathrm{~m}$.
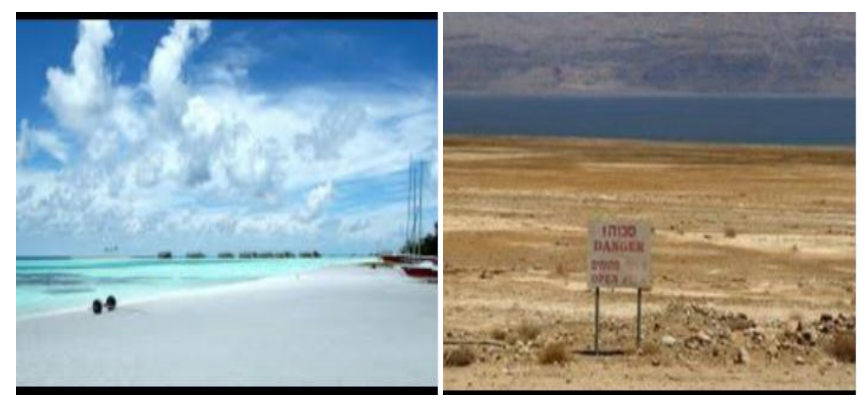

Category 2 - Open terrain with well scattered obstructions having heights generally between $\mathrm{I} .5$ to $10 \mathrm{~m}$.
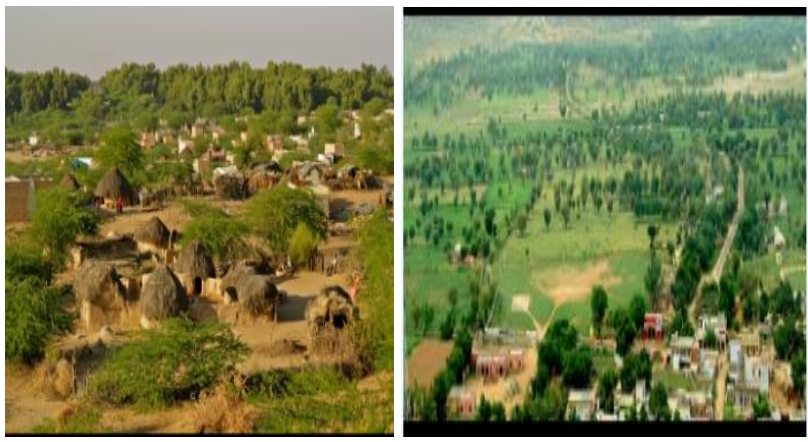

Category 3 - Terrain with numerous closely spaced obstructions having the size of building-structures up to 10 $\mathrm{m}$ in height with or without a few isolated tall structures.
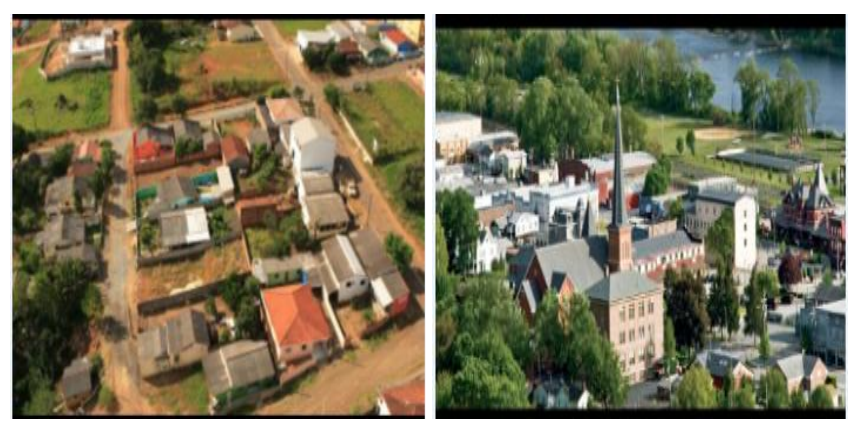

Category 4 - Terrain with numerous large high closely spaced obstructions. 

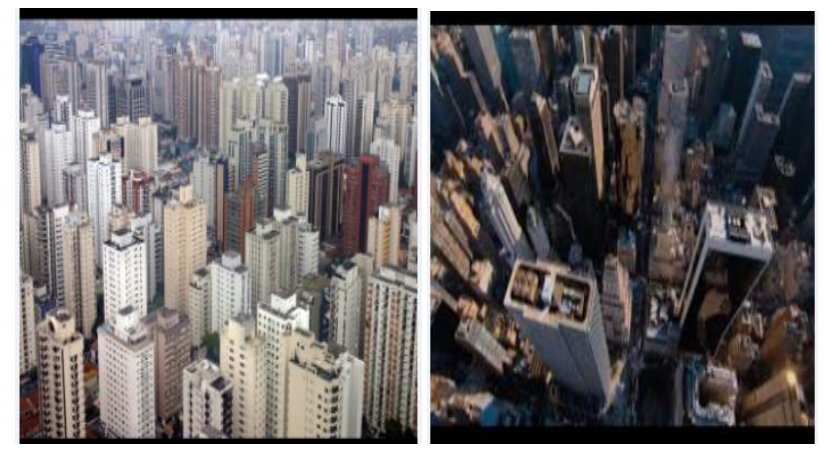

\section{BUILDING DETAILS:}

Three models were prepare I,e (G+10), $(\mathrm{G}+20)$ and $(\mathrm{G}+30)$

\begin{tabular}{|l|l|}
\hline \multicolumn{2}{|l|}{ Model -1: Details } \\
\hline Type of Structure & RCC Frame Structure \\
\hline Number of storey & Eleven $(\mathrm{G}+10)$ \\
\hline Floor to floor height & $3.5 \mathrm{~m}$ \\
\hline Grade of concrete & $\begin{array}{l}\text { M50 for column } \\
\text { M30 for beam \& slab }\end{array}$ \\
\hline Grade of steel & Fe 415 \\
\hline Beam size & $300 * 600 \mathrm{~mm}^{2}$ \\
\hline Column size & $300 * 800 \mathrm{~mm}^{2}$ \\
\hline Slab & $150 \mathrm{~mm}$ \\
\hline
\end{tabular}

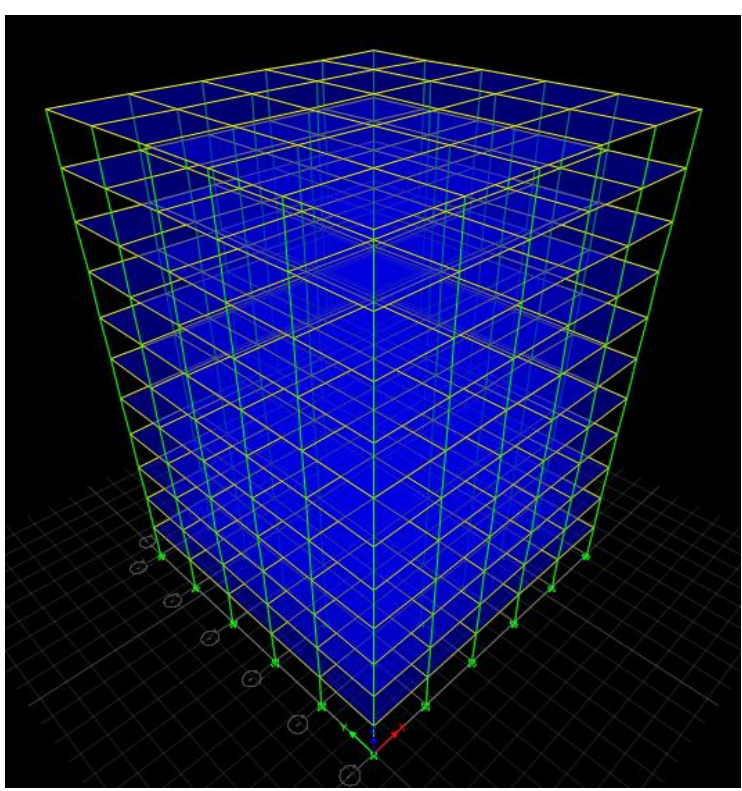

Fig.1. ETABS model of the 11storey building

\begin{tabular}{|l|l|}
\hline \multicolumn{2}{|l|}{ Model -2 :Details } \\
\hline Type of Structure & RCC Frame Structure \\
\hline Number of storey & Twenty one (G+20) \\
\hline Floor to floor height & $3.5 \mathrm{~m}$ \\
\hline
\end{tabular}

\begin{tabular}{|l|l|}
\hline Grade of concrete & $\begin{array}{l}\text { M50 for column } \\
\text { M30 for beam \& slab }\end{array}$ \\
\hline Grade of steel & Fe 415 \\
\hline Beam size & $300 * 600 \mathrm{~mm}^{2}$ \\
\hline Column size & $300 * 800 \mathrm{~mm}^{2}$ \\
\hline Slab & $150 \mathrm{~mm}$ \\
\hline
\end{tabular}

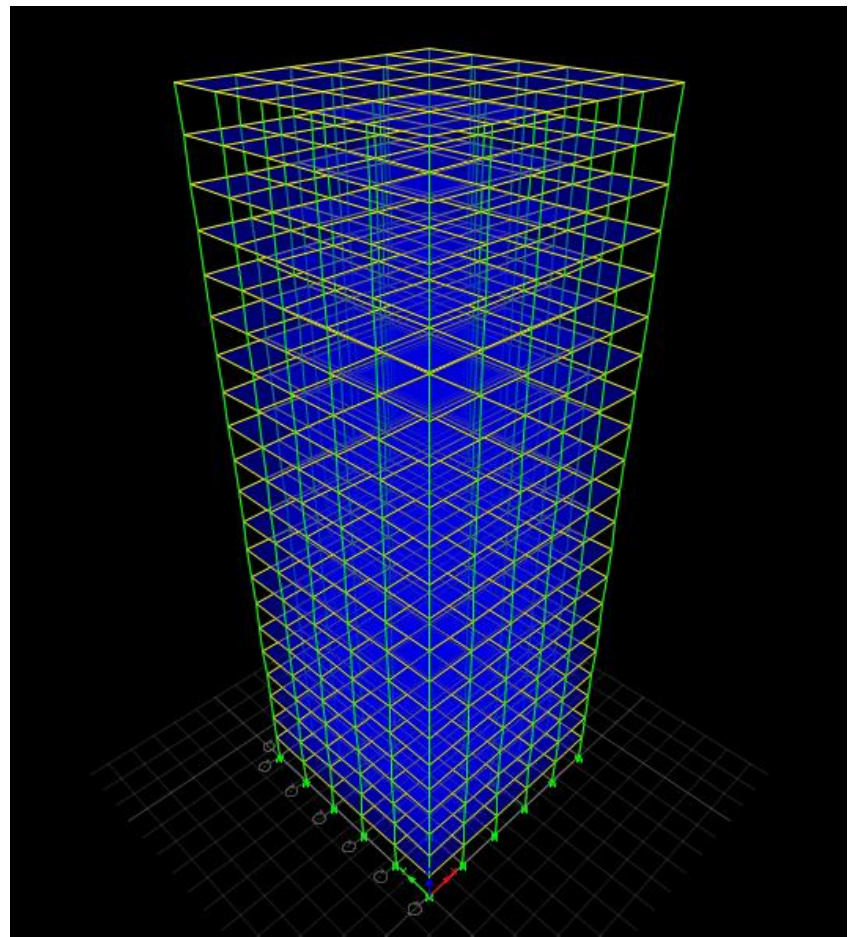

Fig 2 shows the ETABS model of the 21 storey building

\begin{tabular}{|l|l|}
\hline Model -3 Details & RCC Frame Structure \\
\hline Type of Structure & Thirty one (G+30) \\
\hline Number of storey & $3.5 \mathrm{~m}$ \\
\hline Floor to floor height & $\begin{array}{l}\text { M50 for column } \\
\text { M30 for beam \& slab }\end{array}$ \\
\hline Grade of concrete & Fe 415 \\
\hline Grade of steel & $300 * 600 \mathrm{~mm}^{2}$ \\
\hline Beam size & $300 * 800 \mathrm{~mm}^{2}$ \\
\hline Column size & $150 \mathrm{~mm}$ \\
\hline Slab & \\
\hline
\end{tabular}




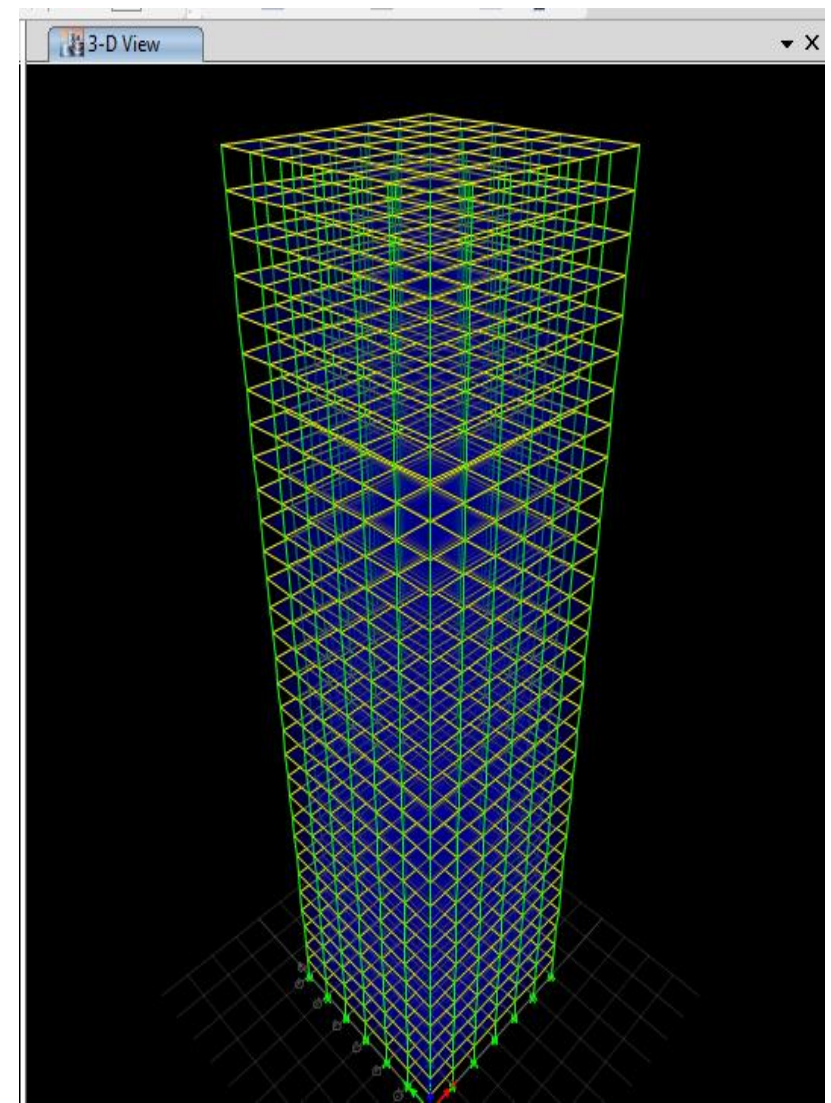

Fig 3: Shows the ETABS model of the 31 storey building

\section{LOADING CONSIDERATION:}

Dead load and live load have been taken as per IS 875 (Part 1) (1987) and IS 875 (Part 2)(1987) respectively. Wind load calculation has been done based on the IS 875 (Part 3) (1987).

\section{Dead Load:}

( IS 875 (Part-1) The loads realized due to the following has been considered by ETABS are

- Self weight of structural members

- Wall load (30cm masonry wall)

- Floor finish

\section{Live Load: ( IS 875 (Part-2) )}

- $\quad$ Live load on floor $=3 \mathrm{kN} / \mathrm{m}^{2}$

- Live load on roof $=1.5 \mathrm{KN} / \mathrm{m}^{2}$

\section{ANALYSIS USING ETABS 2015:}

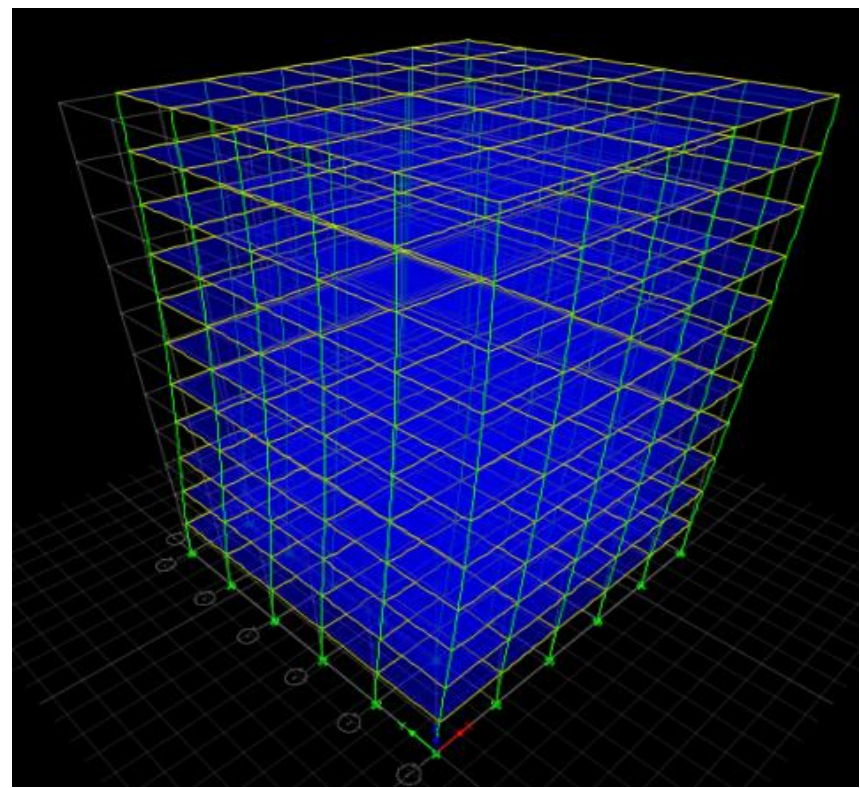

Fig 4 shows the deflection diagram of 11 storey building

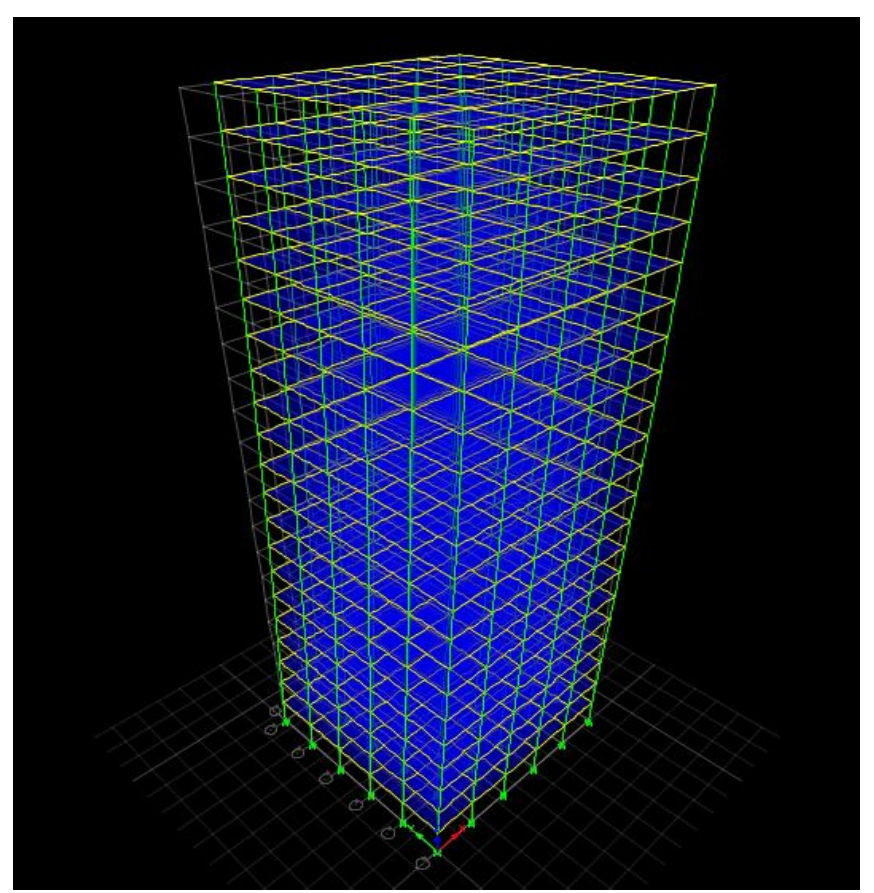

Fig 5 shows the deflection diagram of 21 storey building

\section{Wind load: ( IS 875 (Part-3) )}

- $\quad$ Wind speed $=47 \mathrm{~m} / \mathrm{s}$ 


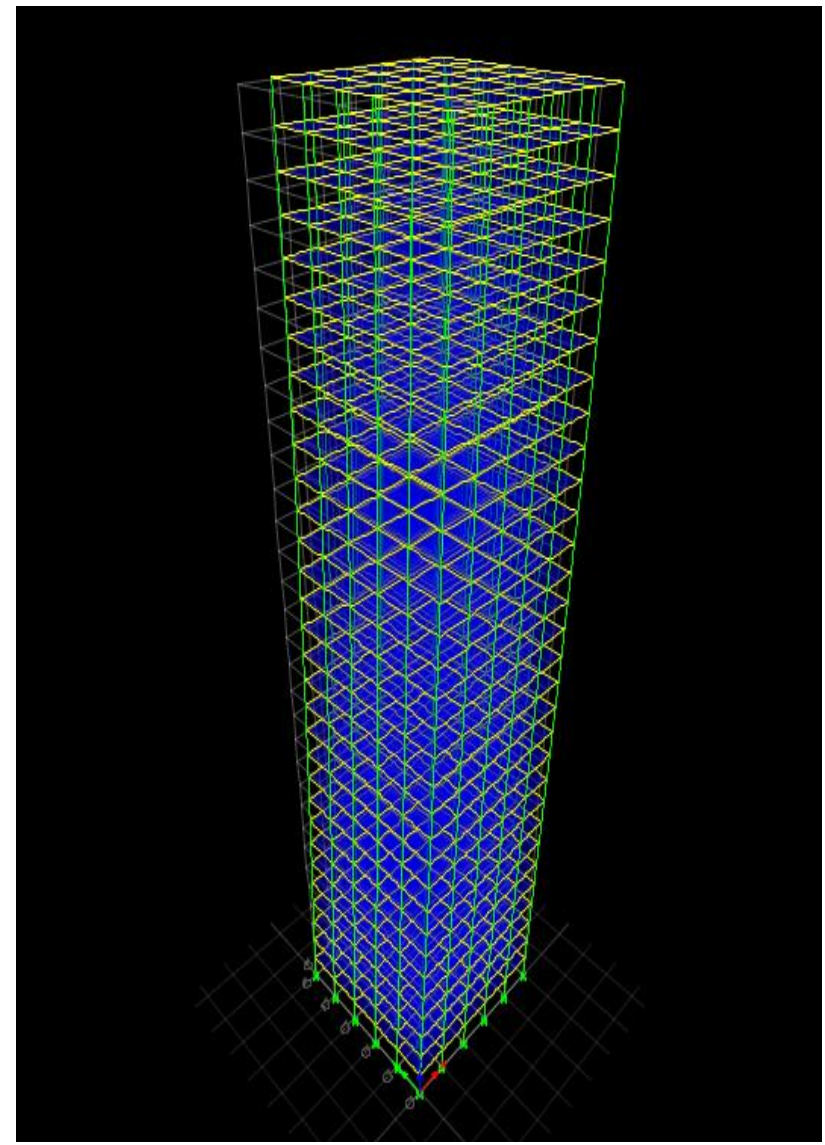

Fig 6 shows the deflection diagram of 31 storey building

\section{RESULTS AND DISCUSSIONS:}

Table 1: Deflection of 11 storey building in different TG

\begin{tabular}{|l|l|l|l|l|l|}
\hline \multicolumn{2}{|l|}{ MODEL-1: } & \multicolumn{3}{|l|}{ Deflection in mm } & \multicolumn{1}{l|}{$\begin{array}{l}\text { TG-1 } \\
\text { v/s }\end{array}$} \\
\cline { 1 - 5 } $\begin{array}{l}\text { Storey } \\
\text { No }\end{array}$ & TG-1 & TG-2 & $\begin{array}{l}\text { TG- } \\
\text { TG- 4 }\end{array}$ & $\begin{array}{l}\text { TG- } \\
4\end{array}$ & T. \\
\hline Storey-11 & 32.1 & 30 & 26 & 19.8 & $38.3 \%$ \\
\hline Storey-10 & 31.6 & 29.5 & 25.7 & 19.5 & $38.3 \%$ \\
\hline Storey-9 & 30.7 & 28.6 & 24.8 & 18.8 & $38.7 \%$ \\
\hline Storey-8 & 29.1 & 27.1 & 23.6 & 17.7 & $39.2 \%$ \\
\hline Storey-7 & 27 & 25.2 & 21.8 & 16.3 & $39.6 \%$ \\
\hline Storey-6 & 24.4 & 22.7 & 19.7 & 14.6 & $40.7 \%$ \\
\hline Storey-5 & 21.3 & 19.8 & 17.1 & 12.7 & $40.37 \%$ \\
\hline Storey-4 & 17.8 & 16.4 & 14.1 & 10.4 & $41.2 \%$ \\
\hline Storey-3 & 13.6 & 12.6 & 10.8 & 8 & $41.2 \%$ \\
\hline
\end{tabular}

\section{Deflection in $\mathrm{mm} \mathrm{v} / \mathrm{s}$ storey level}

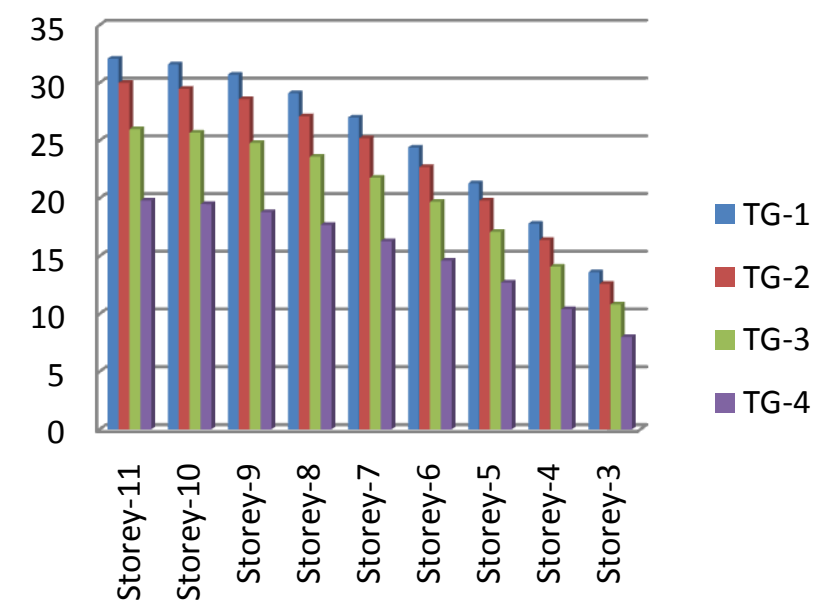

Fig 7 shows Deflection of 11 storey building in different TG

Table 2: Deflection of 21 storey building in different TG

\begin{tabular}{|l|l|l|l|l|l|}
\hline \multicolumn{5}{|l|}{ MODEL-2 Displacement in mm } & \multicolumn{1}{l}{$\begin{array}{l}\text { TG-1 } \\
\text { v/s } \\
\text { TG- 4 }\end{array}$} \\
\cline { 1 - 4 } $\begin{array}{l}\text { Storey- } \\
\text { No }\end{array}$ & TG-1 & TG-2 & TG-3 & TG-4 & \\
\hline $\begin{array}{l}\text { Storey- } \\
21\end{array}$ & 140.9 & 133.6 & 119.5 & 106.4 & $24.5 \%$ \\
\hline $\begin{array}{l}\text { Storey- } \\
20\end{array}$ & 139.7 & 132.5 & 118.4 & 105.5 & $24.48 \%$ \\
\hline $\begin{array}{l}\text { Storey- } \\
19\end{array}$ & 137.9 & 130.8 & 116.9 & 104 & $24.58 \%$ \\
\hline $\begin{array}{l}\text { Storey- } \\
18\end{array}$ & 135.5 & 128.5 & 114.8 & 102.1 & $24.65 \%$ \\
\hline $\begin{array}{l}\text { Storey- } \\
17\end{array}$ & 132.5 & 125.6 & 112.2 & 99.7 & $24.76 \%$ \\
\hline $\begin{array}{l}\text { Storey- } \\
16\end{array}$ & 128.9 & 122.2 & 109.1 & 96.7 & $24.98 \%$ \\
\hline $\begin{array}{l}\text { Storey- } \\
15\end{array}$ & 124.6 & 118.1 & 105.4 & 93.3 & $25.12 \%$ \\
\hline $\begin{array}{l}\text { Storey- } \\
14\end{array}$ & 119.8 & 113.5 & 101.3 & 89.4 & $25.37 \%$ \\
\hline $\begin{array}{l}\text { Storey- } \\
13\end{array}$ & 114.4 & 108.3 & 96.6 & 85.1 & $25.6 \%$ \\
\hline $\begin{array}{l}\text { Storey- } \\
12\end{array}$ & 108.3 & 102.6 & 91.4 & 80.3 & $25.85 \%$ \\
\hline $\begin{array}{l}\text { Storey- } \\
11\end{array}$ & 101.8 & 96.3 & 85.6 & 75.1 & $26.23 \%$ \\
\hline $\begin{array}{l}\text { Storey- } \\
10\end{array}$ & 94.6 & 89.5 & 79.7 & 69.4 & $26.6 \%$ \\
\hline Storey-9 & 87 & 82.2 & 73.1 & 63.5 & $27 \%$ \\
\hline Storey-8 & 78.8 & 74.4 & 66.1 & 57.1 & $27.5 \%$ \\
\hline Storey-7 & 70.1 & 66.2 & 58.7 & 50.5 & $27.96 \%$ \\
\hline Storey-6 & 60.9 & 57.4 & 50.9 & 43.5 & $28.57 \%$ \\
\hline Storey-5 & 51.3 & 48.3 & 42.8 & 36.5 & $28.85 \%$ \\
\hline Storey-4 & 41.2 & 38.8 & 34.3 & 29.1 & $29.37 \%$ \\
\hline Storey-3 & 30.7 & 28.9 & 25.5 & 21.6 & $29.64 \%$ \\
\hline
\end{tabular}




\section{Deflection in $\mathrm{mm} v / \mathrm{s}$ Storey level}

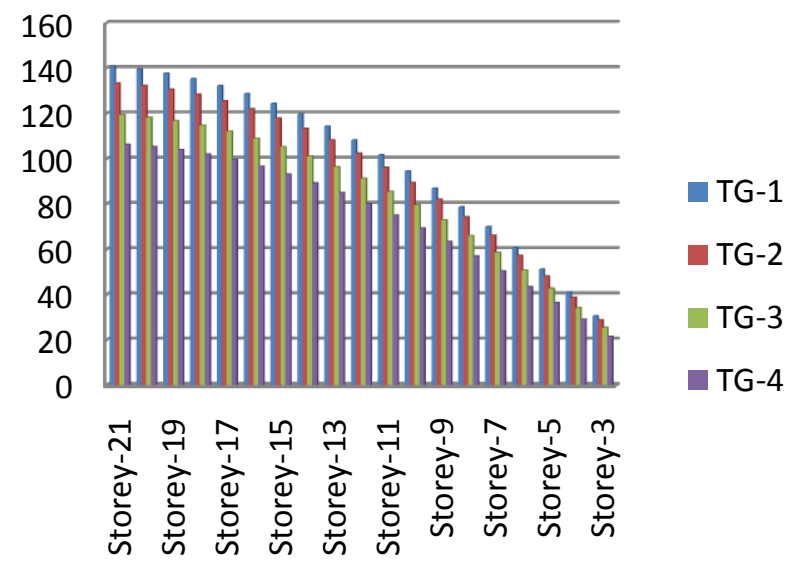

Fig 8 shows Deflection of 21 storey building in different TG

Table 3: Deflection of 31 storey building in different TG

\begin{tabular}{|l|l|l|l|l|l|}
\hline \multicolumn{2}{|l|}{ MODEL-3 } & \multicolumn{3}{|c|}{ Deflection in mm } & \multicolumn{1}{l}{$\begin{array}{l}\text { TG-1 } \\
\text { va- }\end{array}$} \\
\hline Storey No & TG-1 & TG-2 & TG-3 & TG-4 & TG- \\
\hline Storey-31 & 357.6 & 342.6 & 310.9 & 289.9 & $18.9 \%$ \\
\hline Storey-30 & 354.7 & 339.8 & 308.3 & 287.5 & $18.95 \%$ \\
\hline Storey-29 & 351.2 & 336.4 & 305.2 & 284.5 & $18.99 \%$ \\
\hline Storey-28 & 347 & 332.4 & 301.5 & 281 & $19.02 \%$ \\
\hline Storey-27 & 342.1 & 327.7 & 297.2 & 276.9 & $19.05 \%$ \\
\hline Storey-26 & 336.6 & 322.4 & 292.3 & 272.2 & $19.13 \%$ \\
\hline Storey-25 & 330.5 & 316.4 & 286.9 & 267 & $19.2 \%$ \\
\hline Storey-24 & 323.6 & 309.8 & 280.9 & 261.2 & $19.28 \%$ \\
\hline Storey-23 & 316.2 & 302.6 & 274.3 & 254.9 & $19.38 \%$ \\
\hline Storey-22 & 308 & 294.8 & 267.1 & 248.1 & $19.45 \%$ \\
\hline Storey-21 & 299.3 & 286.4 & 259.4 & 240.7 & $19.58 \%$ \\
\hline Storey-20 & 290 & 277.4 & 251.2 & 232.8 & $19.72 \%$ \\
\hline Storey-19 & 280 & 267.8 & 242.4 & 224.5 & $19.82 \%$ \\
\hline Storey-18 & 269.4 & 257.6 & 233.1 & 215.6 & $19.97 \%$ \\
\hline Storey-17 & 258.4 & 246.9 & 223.3 & 206.3 & $20.16 \%$ \\
\hline Storey-16 & 246.5 & 235.6 & 213 & 196.5 & $20.28 \%$ \\
\hline Storey-15 & 234.2 & 223.8 & 202.2 & 186.2 & $20.49 \%$ \\
\hline Storey-14 & 221.4 & 211.4 & 191 & 175.5 & $20.7 \%$ \\
\hline Storey-13 & 208 & 198.6 & 179.3 & 164.4 & $20.96 \%$ \\
\hline Storey-12 & 194.1 & 185.3 & 167.2 & 153 & $21.17 \%$ \\
\hline Storey-11 & 179.7 & 171.4 & 154.6 & 141.1 & $21.48 \%$ \\
\hline Storey-10 & 164.8 & 157.2 & 141.7 & 128.9 & $21.78 \%$ \\
\hline Storey-9 & 149.5 & 142.5 & 128.3 & 116.4 & $22.14 \%$ \\
\hline Storey-8 & 133.7 & 127.4 & 114.7 & 103.7 & $22.44 \%$ \\
\hline Storey-7 & 117.5 & 111.9 & 100.7 & 90.7 & $22.81 \%$ \\
\hline Storey-6 & 101 & 96.1 & 86.4 & 77.5 & $23.27 \%$ \\
\hline Storey-5 & 84.1 & 80 & 71.8 & 64.2 & $23.66 \%$ \\
\hline Storey-4 & 66.9 & 63.5 & 57 & 50.8 & $24.07 \%$ \\
\hline Storey-3 & 49.4 & 46.9 & 42 & 37.4 & $24.29 \%$ \\
\hline
\end{tabular}

\section{Deflection in $\mathrm{mm} \mathrm{v} / \mathrm{s}$ storey level}

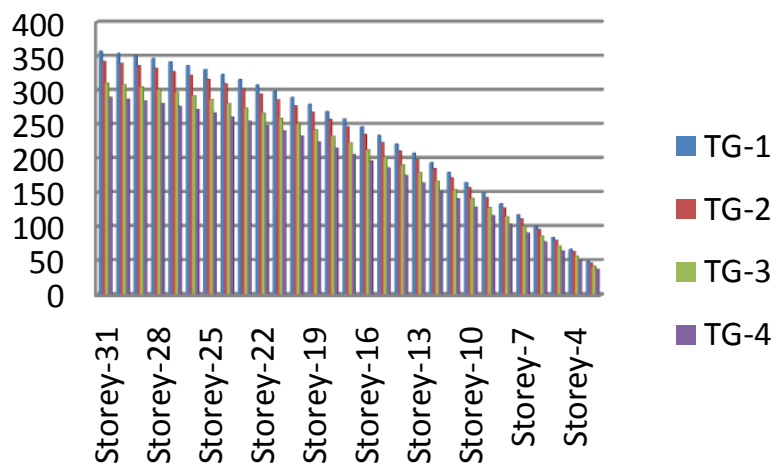

Fig 9 shows Deflection of 31 storey building in different TG

\section{CONCLUSION}

$>$ As the height of the model increases, deflection on top storey also increases

$>$ Due to wind load, Deflection on model-3 is more than model-2 and model-1 as shown in Fig-7,8, and 9

$>$ In Model-1: Deflection in TG-1 is 6\%, 19\% and $38.3 \%$ more than TG-2, TG-3, and TG-4 on top storey.

$>$ In Model-2: Deflection in TG-1 is $5 \%, 15 \%$ and $24.5 \%$ more than TG-2, TG-3, and TG-4 on top storey.

$>$ In Model-3: Deflection in TG-1 is $4 \%, 13 \%$ and 18.9\% more than TG-2, TG-3, and TG-4 on top storey.

$>\quad$ But in all 3 models \% $\%^{\text {age }}$ deflection between TG-1 and TG-4 is maximum at $3^{\text {rd }}$ storey .

\section{REFRENCES}

[1] IS: 875:1987 (part-1 and part2) "Indian Standard Code of practice for design loads", Bureau of Indian Standards, New Delhi

[2] IS: 875:1987 (part-3 ) "Indian Standard Code of practice for design Wind loads", Bureau of Indian Standards, New Delhi

[3] Abdur Rahman, Saiada Fuadi Fancy, Shamim Ara Bobby, Analysis of drift due to wind loads and earthquake loads on tall structures by programming language $C$, International Journal of Scientific and Engineering Research, Vol. 3, Issue 6, June 2012.

[4] B. Dean Kumar and B.L.P. Swami, Wind effects on tall building frames-influence of dynamic parameters, Indian Journal of Science and Technology, Vol. 3, No. 5.May 2010, 583-587.

[5] T. Kijewski and A. Kareem, Full-scale study of the behavior of tall buildings under winds, NatHaz Modeling Laboratory, Department of Civil Engineering and Geological Sciences, University of Notre Dame, Notre Dame, IN 46556. 